\title{
Stability Analysis Using Controlled Invariant Sets for Piecewise-Affine
}

\section{Systems}

\author{
Luis Rodrigues \\ Assistant Professor \\ Department of Mechanical and Industrial Engineering \\ Concordia University \\ 2160B Bishop Street, B-304 \\ Montréal, QC H3G 2E9, Canada \\ Email: luisrod@me.concordia.ca
}

July 16, 2003

\begin{abstract}
This paper presents new stability conditions for closed-loop piecewise-affine (PWA) systems. The result is based on controlled invariant sets for PWA systems, which are defined by extending the notion of semi-ellipsoidal invariant sets for constrained linear systems reported in previous research. The paper shows that by proper use of the control input, concatenations of semi-ellipsoidal sets can be made invariant for the trajectories of PWA systems. Furthermore, based on these controlled invariant sets, the paper presents a result for stability of a closed-loop PWA system which is less conservative than existing approaches in the literature. In this result it is shown that a PWA system is stable inside the intersection of any level set for a local Lyapunov function and the design set where the function is defined, provided the flow points inwards at the boundaries of the intersection. This result is less conservative than previous approaches and it enables the designer to have an estimation of a much larger region of exponential stability then it would be possible using previous results. A numerical example is presented in which is made clear by comparison with previous approaches that the estimated region of stability can be made significantly larger using the new stability conditions developed in this paper.
\end{abstract}

Keywords: piecewise-affine systems, invariant sets, tangent cone, ellipsoids, Lyapunov function.

\section{Introduction}

Invariant sets in control of dynamical systems are an important notion because they enable a designer to guarantee that state trajectories starting inside them will remain inside a given desirable set. Examples of desirable sets are: a stability region, a safety set or a set corresponding to control and state constraints. 
The study of invariant sets in control has been a very active area of research. Blanchini [1] offers a detailed survey of this area up until 1999. Earlier contributions with connections to piecewise-affine systems were devoted to constrained linear systems [4, 15]. Gutman and Cwikel [4] have shown that for discrete-time linear systems with the control and state constrained to convex polyhedra, some control laws verifying the constraints were piecewise linear. For constrained linear systems, O'Dell and Misawa [15] have defined the notion of semi-ellipsoidal set as the intersection of a controlled invariant ellipsoid and state constraints. They have investigated semi-ellipsoidal invariant sets as possible solutions to the maximal viability set for continuous-time linear systems with a bounded input and the state constrained to convex polyhedra. Invariant sets have also been used to solve the reachability problem for verification of hybrid systems $[6,17]$ where the continuous dynamics of the hybrid systems are either of very simple form (constant derivative) or are a simple abstraction (rectangular differential inclusions) of more complicated dynamics. A similar concept to invariant set, maximal safety set, has also been studied in the literature by Sastry et.al. for a class of discrete-time linear hybrid systems [24] and for a class of continuous-time linear hybrid systems [23]. Robust controlled invariant sets for a class of uncertain hybrid systems has been investigated by Lin and Antsaklis in [12] and the maximal controlled invariant set of switched linear systems has been studied by Julius and van der Schaft in [9].

Although an active area of research, very limited work has concentrated on invariant sets for either continuous-time PWA systems $[7,22]$ or discrete-time PWA systems $[10,13]$. The first attempt to define invariant sets for PWA systems appears to have been under the framework of linear hybrid systems [7]. Jirstrand [7] has defined classes of invariant sets for PWA systems with each local dynamics restricted to be nonsingular and to have a real eigenvalue. The invariant sets were formed as concatenations of ellipsoids, paraboloids and quadratic cones. An iterative update algorithm for computation of these sets is presented but it is not shown that it has a fixed point. Therefore, although an important first step, convergence of the algorithm and decidability of the formulated problem are not guaranteed. Roll [22] presented an approach to investigate invariance of approximating automata for continuous-time PWA systems to changes in the dynamics and translation of the switching hyperplanes. The flow at the boundaries of the switching hyperplanes has been used to develop conditions for invariance in the three possible cases: the flow points always outwards, the flow points always inwards, and there is at least one point where the flow is parallel to the boundaries. When only the dynamics or the switching boundaries vary, the solution set is convex. Unfortunately, when both the dynamics and the switching surfaces change, the conditions lead to a non-convex solution set that is hard to efficiently represent. A finite union of polytopes was shown to be a robustly controllable set for constrained discrete-time PWA systems by Kerrigan and Mayne in [10]. Although the authors present an interesting formulation of the problem, as pointed out in [10], algorithms based directly on the results presented there might be too inefficient to be realizable for large or complex systems, which motivates further research. A technique for receding horizon control for discrete-time piecewise-linear systems based on invariant sets has been reported by Mukai et. al. in [13]. The proposed control strategy is implemented by solving a min-max type optimization problem at each time step.

As seen in the last paragraph, previous research on invariant sets for PWA systems has been very limited 
and, except for references [7, 22], it has been geared towards discrete-time systems. Furthermore, it has not been concerned with the important problem of estimation of regions of exponential stability for systems under piecewise-affine control. The powerful connection between invariant sets and Lyapunov functions has also not been explored to estimate larger regions of attraction for the closed-loop trajectories. The current work tries to fill this gap based on previous work of the author, which addressed synthesis methods for PWA systems [18], for a PWA approximation of a class of nonlinear systems [19] and for classes of nonlinear [20] and hybrid systems [21]. The current paper builds on this research and presents a less conservative result for the stability of a closed-loop continuous-time PWA system. The approach given in the paper is based on a concatenation of semi-ellipsoidal sets [15] that are shown to be controlled invariant sets for PWA systems. The result presented here enables the designer to have an estimation of a larger region of exponential stability than it would be possible using previous results for PWA systems [18]. Since the paper will derive stability conditions for PWA systems based on Lyapunov theory, we start by reviewing Lyapunov stability results for closed-loop PWA systems under state feedback. Next, for the same closedloop PWA systems, a new and less conservative stability result is presented based on controlled invariant sets. The important improvements enabled by this new result relative to the estimation of the size of the region of guaranteed exponential stability are then illustrated in a numerical example. It should be mentioned that although state feedback closed-loop systems were selected for illustrative purposes, the new result can be used for any controlled PWA system. Finally, the paper finishes by presenting the conclusions

\section{Lyapunov Stability Results for Piecewise-Affine Systems}

It is assumed that a PWA system and a corresponding partition of the state space with polytopic cells $\mathcal{R}_{i}$, $i \in \mathcal{I}=\{1, \ldots, M\}$ are given (see [19] for generating such a partition). Following [8, 16, 5], each cell is constructed as the intersection of a finite number $\left(p_{i}\right)$ of half spaces

$$
\mathcal{R}_{i}=\left\{x \mid H_{i}^{T} x-g_{i}<0\right\}
$$

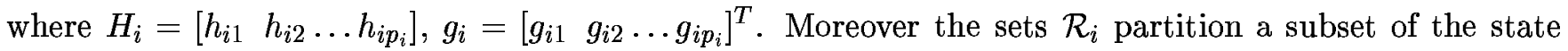
space $\mathcal{X} \subset \mathbb{R}^{n}$ such that $\cup_{i=1}^{M} \overline{\mathcal{R}}_{i}=\mathcal{X}, \mathcal{R}_{i} \cap \mathcal{R}_{j}=\emptyset, i \neq j$, where $\overline{\mathcal{R}}_{i}$ denotes the closure of $\mathcal{R}_{i}$. Within each cell the dynamics are affine of the form

$$
\dot{x}(t)=A_{i} x(t)+b_{i}+B_{i} u(t)
$$

where $x(t) \in \mathbb{R}^{n}$ and $u(t) \in \mathbb{R}^{m}$. Any two cells sharing a common facet will be called level-1 neighboring cells. Let $\mathcal{N}_{i}=\left\{\right.$ level-1 neighboring cells of $\left.\mathcal{R}_{i}\right\}$. It is assumed that vectors $c_{i j} \in \mathbb{R}^{n}$ and scalars $d_{i j}$ exist such that the facet boundary between cells $\mathcal{R}_{i}$ and $\mathcal{R}_{j}$ is contained in the hyperplane described by $\left\{x \in \mathbb{R}^{n} \mid c_{i j}^{T} x-d_{i j}=0\right\}$, for $i=1, \ldots, M, j \in \mathcal{N}_{i}$. A parametric description of the boundaries can then be obtained as [5]

$$
\overline{\mathcal{R}_{i}} \cap \overline{\mathcal{R}_{j}} \subseteq\left\{l_{i j}+F_{i j} s \mid s \in \mathbb{R}^{n-1}\right\}
$$


for $i=1, \ldots, M, j \in \mathcal{N}_{i}$, where $F_{i j} \in \mathbb{R}^{n \times(n-1)}$ (full rank) is the matrix whose columns span the null space of $c_{i j}$, and $l_{i j} \in \mathbb{R}^{n}$ is given by $l_{i j}=c_{i j}\left(c_{i j}^{T} c_{i j}\right)^{-1} d_{i j}$. For a definition of trajectories or solutions for system (2) see [18].

Following prior analysis of PWA systems $[8,16,5]$, consider the piecewise-quadratic Lyapunov function continuous at the boundaries and defined in $\cup_{i=1}^{M} \mathcal{R}_{i}$ by the expression

$$
\begin{gathered}
V(x)=\sum_{i=1}^{M} \beta_{i}(x) V_{i}(x), V(x)>0 \\
V_{i}(x)=\left(x^{T} P_{i} x+2 q_{i}^{T} x+r_{i}\right)
\end{gathered}
$$

where $P_{i}=P_{i}^{T} \in \mathbb{R}^{(n \times n)}, q_{i} \in \mathbb{R}^{n}, r_{i} \in \mathbb{R}$ and

$$
\beta_{i}(x)=\left\{\begin{array}{ll}
1, & x \in \mathcal{R}_{i} \\
0, & x \in \mathcal{R}_{j}, j \neq i
\end{array},\right.
$$

for $i=1, \ldots, M$. The expression for the candidate Lyapunov function in each region can be recast as [8]

$$
V_{i}(x)=\left[\begin{array}{c}
x \\
1
\end{array}\right]^{T}\left[\begin{array}{cc}
P_{i} & q_{i} \\
q_{i}^{T} & r_{i}
\end{array}\right]\left[\begin{array}{l}
x \\
1
\end{array}\right]=\bar{x}^{T} \overline{P_{i}} \bar{x}
$$

Let $\alpha_{i}$ be the desired decay rate for this Lyapunov function in each region $\mathcal{R}_{i}$. Then, we define the performance criterion

$$
\mathcal{J}=\min _{i=1 \ldots M} \alpha_{i}
$$

Using a PWA control signal $u=K_{i} x+m_{i}$, the closed-loop state equations in each region $\mathcal{R}_{i}$ are

$$
\dot{x}=\left(A_{i}+B_{i} K_{i}\right) x+\left(b_{i}+B_{i} m_{i}\right) \equiv \bar{A}_{i} x+\bar{b}_{i} .
$$

Setting $x_{c l}^{i}$ to be the closed-loop equilibrium point for region $\mathcal{R}_{i}$ yields

$$
\left(A_{i}+B_{i} K_{i}\right) x_{c l}^{i}+\left(b_{i}+B_{i} m_{i}\right)=0 .
$$

Using the boundary description (3), continuity of the candidate Lyapunov function across the boundaries is enforced for each region $\mathcal{R}_{i}$ and for $j \in \mathcal{N}_{i}$ by [5]

$$
\begin{aligned}
F_{i j}^{T}\left(P_{i}-P_{j}\right) F_{i j} & =0, \\
F_{i j}^{T}\left(P_{i}-P_{j}\right) l_{i j}+F_{i j}^{T}\left(q_{i}-q_{j}\right) & =0 \\
l_{i j}^{T}\left(P_{i}-P_{j}\right) l_{i j}+2\left(q_{i}-q_{j}\right)^{T} l_{i j}+\left(r_{i}-r_{j}\right) & =0 .
\end{aligned}
$$

Remark 1 Note that because $V(x)>0$ (defined in $\mathbb{R}^{n}$ ) is continuous, the fact that $V$ is piecewise- 
quadratic also implies that $V$ is radially unbounded, i.e., $V(x) \rightarrow+\infty$ as $\|x\| \rightarrow \infty$, provided $P_{i}>0$, $i=1, \ldots, M$.

The function $V(x)$ in (4) will be a Lyapunov function with a decay rate of $\alpha_{i}$ for region $\mathcal{R}_{i}$ if, for fixed $\epsilon \geq 0$,

$$
x \in \mathcal{R}_{i} \Rightarrow\left\{\begin{array}{l}
V_{i}(x)>\epsilon\left\|x-x_{c l}\right\|_{2}, \\
\frac{d}{d t} V_{i}(x)<-\alpha_{i} V_{i}(x) .
\end{array}\right.
$$

where $x_{c l}$ is the desired closed-loop equilibrium point of the system. Using the polytopic description of the cells (1) and the $\mathcal{S}$-procedure [2], it can be shown [21] that conditions (11) are implied by the existence of $P_{i}=P_{i}^{T}>0, q_{i}, r_{i}$, and matrices $Z_{i}$ and $\Lambda_{i}$ with nonnegative entries satisfying

$$
\begin{gathered}
{\left[\begin{array}{cc}
\left(P_{i}-\epsilon I_{n}-\bar{H}_{i}^{T} Z_{i} \bar{H}_{i}\right) & \left(q_{i}+\epsilon x_{c l}+\bar{H}_{i}^{T} Z_{i} \bar{g}_{i}\right) \\
(\cdot)^{T} & r_{i}-\epsilon x_{c l}^{T} x_{c l}-\bar{g}_{i}^{T} Z_{i} \bar{g}_{i}
\end{array}\right]>0,} \\
{\left[\begin{array}{cc}
\left(\bar{A}_{i}^{T} P_{i}+P_{i} \bar{A}_{i}+\bar{H}_{i}^{T} \Lambda_{i} \bar{H}_{i}+\alpha_{i} P_{i}\right)\left(P_{i} \bar{b}_{i}+\bar{A}_{i}^{T} q_{i}-\bar{H}_{i}^{T} \Lambda_{i} \bar{g}_{i}+\alpha_{i} q_{i}\right) \\
(\cdot)^{T} & 2 \bar{b}_{i}^{T} q_{i}+\bar{g}_{i}^{T} \Lambda_{i} \bar{g}_{i}+\alpha_{i} r_{i}
\end{array}\right]<0 .}
\end{gathered}
$$

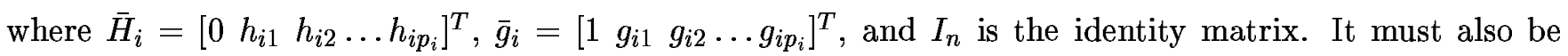
ensured that the trajectories do not stay at a switching boundary in a sliding mode. Reference [18] shows that constraints for avoidance of sliding modes are

$$
\begin{aligned}
c_{i j}^{T}\left(A_{i}+B_{i} K_{i}-A_{j}-B_{j} K_{j}\right) F_{i j} & =0 \\
c_{i j}^{T}\left[\left(A_{i}+B_{i} K_{i}-A_{j}-B_{j} K_{j}\right) l_{i j}+b_{i}+B_{i} m_{i}-b_{j}-B_{j} m_{j}\right] & =0
\end{aligned}
$$

for $i=1, \ldots, M$ and $j \in \mathcal{N}_{i}$.

Definition 2.1 The state feedback synthesis optimization problem is

$$
\begin{aligned}
\max & \min _{i} \alpha_{i} \\
\text { s.t. } & (9), \quad(10), \quad(12), \quad(13), \quad(14) \\
& Z_{i} \succ 0, \Lambda_{i} \succ 0, \alpha_{i}>l_{0} \geq 0, \\
& -l_{1} \prec K_{i} \prec l_{1}, \quad-l_{2} \prec m_{i} \prec l_{2},
\end{aligned}
$$

$i=1, \ldots, M$, where $\succ, \prec$ mean component-wise inequalities, $l_{0}$ is a scalar bound and $l_{1}, l_{2}$ are vector bounds. Note that the optimization variables are $x_{c l}^{i}, K_{i}, m_{i}, \alpha_{i}, P_{i}, q_{i}, r_{i}, Z_{i}$ and $\Lambda_{i}$.

To simplify the optimization problem, the desired closed-loop equilibrium points for each polytopic region, $x_{c l}^{i}$, are selected a-priori using the optimization algorithm described in [19]. Then, expression (13) is called a bilinear matrix inequality (BMI) [3]. Suboptimal solutions to the optimization problem can be obtained with the algorithms presented in [18] 
Theorem 2.1 Assume the Lyapunov function (4) is defined in $\mathcal{X} \subseteq \mathbb{R}^{n}$. If there is a solution to the design problem from Definition 2.1, the closed-loop system is locally asymptotically stable inside any subset of the largest level set of the control Lyapunov function (4) that is fully contained in $\mathcal{X}$. If $\epsilon>0$ then the convergence is exponential. If, furthermore, $\mathcal{X}=\mathbb{R}^{n}$ then the exponential stability is global.

Proof: See [18]

\section{Stability of the Closed-Loop PWA System}

In this section, a new stability result that is less conservative than Theorem 2.1 will be presented. The result is based on the notion of controlled invariant set.

Definition 3.1 [1] $A$ set $\mathcal{C}$ is (positively) invariant for a dynamical system if once the state $x(t)$ enters that set at any given time $t_{0}$, it remains in it for all future time, i.e, $x\left(t_{0}\right) \in \mathcal{C} \Rightarrow x(t) \in \mathcal{C}, \forall_{t \geq t_{0}} . A$ controlled invariant set is a set that can be made positively invariant by a proper choice of a control action.

Stability will be defined within a set called the design set [18]. One important subclass of design sets to be considered in this work is defined next.

Definition $3.2[18] A$ design set $\mathcal{D}$ is a nonempty closed subset of the domain of the state variable $x$.

Definition $3.3 A$ design set $\mathcal{D}$ is called a polytopic design set if:

1. $\mathcal{D} \subseteq \mathcal{X}$ where $\mathcal{X}=\cup_{i=1}^{M} \overline{\mathcal{R}}_{i}$

2. $\mathcal{D}$ is the intersection of a finite number of half spaces $\mathcal{D}_{j}=\left\{x \mid c_{j}^{T} x \leq d_{j}\right\}$ in the form

$$
\mathcal{D}=\left\{x \mid c_{j}^{T} x \leq d_{j}, j=1, \ldots, p\right\}
$$

The main result in this section states formally that a closed-loop PWA system is stable inside the intersection of any level set for a local Lyapunov function and the design set where the function is defined, provided the flow points inwards at the boundaries of the intersection. To prove this result we need the following definition and Lemma.

Definition 3.4 [11] Given a real number $\gamma>0$, a $\gamma$-level set of a Lyapunov function $V$ is the set $\{x \in$ $\left.\mathbb{R}^{n} \mid V(x) \leq \gamma\right\}$. 
Remark 2 Note that the $\gamma$-level set of the $k$ th sector (corresponding to region $\mathcal{R}_{k}$ ) of the Lyapunov function (4) is invariant under the flow $\dot{x}(t)=\bar{A}_{k} x(t)+\bar{b}_{k}$. This invariant set is described by the ellipsoid

$$
\varepsilon_{\gamma}^{k}=\left\{x \in \mathbb{R}^{n} \mid\left(x-x_{c l}^{k}\right)^{T} P_{k}\left(x-x_{c l}^{k}\right)+r_{k} \leq \gamma\right\} .
$$

Lemma 3.1 [14] Consider the system $\dot{x}(t)=f(x(t))$ and assume that, for each initial condition in a set $\mathcal{D}$, it admits a globally unique solution. Let $\mathcal{L} \subseteq \mathcal{D}$ be a closed and convex set and let $\partial_{\mathcal{L}}$ be its boundary. Then the set $\mathcal{L}$ is positively invariant for the system if and only if $f(x) \in \mathcal{C}_{\mathcal{L}}(x)$ for all $x \in \partial_{\mathcal{L}}$, where $\mathcal{C}_{\mathcal{L}}(x)$ is the tangent cone for the set $\mathcal{L}$ at the point $x$.

Remark 3 Roughly speaking, the above Theorem just states that for a set to be positively invariant, the vector field has to point inwards at the boundaries of the set.

Remark 4 For the boundary of smooth sets $\mathcal{L}$ the tangent cone $\mathcal{C}_{\mathcal{L}}(x)$ at the point $x$ is just the half space tangent to $\mathcal{L}$ at $x$ shifted to the origin. Thus, the tangent cones for the boundaries of the ellipsoid $\varepsilon_{\gamma}^{k}$ and design set constraints $\mathcal{D}_{j}$ are $\mathcal{C}_{\varepsilon_{\gamma}^{k}}=\left\{y \mid\left(x-x_{c l}^{k}\right)^{T} P_{k} y \leq 0\right\}$ and $\mathcal{C}_{\mathcal{D}_{j}}=\left\{y \mid c_{j}^{T} y \leq 0\right\}$, respectively. Note that, as expected, the orientation of the boundary of $\mathcal{C}_{\mathcal{D}_{j}}(x)$ is fixed and depends only on $c_{j}$. It does not depend on the point $x$.

The two sets defined in (16) will be needed in part 2 of the proof of Theorem 3.1. That part of the proof follows closely the one presented in [15] for a related Theorem relative to constrained linear systems.

$$
\begin{aligned}
\partial_{\dot{\mathcal{D}}_{j}} & \equiv\left\{x \mid c_{j}^{T} \dot{x}=0\right\} \\
\dot{\mathcal{D}}_{j}^{+} & \equiv\left\{x \mid c_{j}^{T} \dot{x}>0\right\}
\end{aligned}
$$

Theorem 3.1 Let the design set $\mathcal{D}$ be polytopic as in definition 3.3 and let $\partial_{\mathcal{D}_{j}}$ be the boundary of $\mathcal{D}_{j}$ and $\partial_{\mathcal{D}}$ be the boundary of $\mathcal{D}$. Assume there exits a parametric description for $\partial_{\mathcal{D}_{j}}, j=1, \ldots, p$ of the form

$$
x \in \partial_{\mathcal{D}_{j}} \subseteq \mathcal{H}_{j}=\left\{v_{j}+T_{j} s \mid s \in \mathbb{R}^{n-1}\right\}
$$

where all involved matrices have appropriate dimensions. Given $\gamma>0$ define the closed set $\varepsilon_{\gamma}=$ $\cup_{k=1}^{M}\left(\varepsilon_{\gamma}^{k} \cap \overline{\mathcal{R}_{k}}\right)$ and the closed subset $\tilde{\varepsilon}_{\gamma}=\varepsilon_{\gamma} \cap \mathcal{D}$. If there is a solution to the controller synthesis problem from definition 2.1 then the following are verified:

1. The set $\varepsilon_{\gamma}$ is a $\gamma$-level set of the Lyapunov function (4). As such, $\varepsilon_{\gamma}$ is an invariant set for the PWA system for all $\gamma>0$ such that $\varepsilon_{\gamma} \subseteq \mathcal{D}$ and the closed-loop system is exponentially stable inside all such $\varepsilon_{\gamma}$; 
2. The set $\tilde{\varepsilon}_{\gamma}$ is an invariant set of the system if and only if $\tilde{\varepsilon}_{\gamma} \cap \partial_{\mathcal{D}_{j}} \cap \dot{\mathcal{D}_{j}^{+}}=\emptyset, j=1, \ldots, p$,

3. The closed-loop system is exponentially stable inside $\tilde{\varepsilon}_{\gamma}$ for any $\gamma>0$ for which there are $\epsilon_{k j}>0$ such that $\forall_{(j, k)} \mid \overline{\mathcal{R}_{k}} \cap \mathcal{H}_{j} \neq \emptyset$, expression (18) is verified.

$$
\left[\begin{array}{cc}
T_{j}^{T} P_{k} T_{j} & T_{j}^{T} P_{k}\left(v_{j}-x_{c l}^{k}\right)-\epsilon_{k j} T_{j}^{T} A_{k}^{T} c_{j} \\
\left(T_{j}^{T} P_{k}\left(v_{j}-x_{c l}^{k}\right)-\epsilon_{k j} T_{j}^{T} A_{k}^{T} c_{j}\right)^{T} & \left(v_{j}-x_{c l}^{k}\right)^{T} P_{k}\left(v_{j}-x_{c l}^{k}\right)+r_{k}-\gamma-2 \epsilon_{k j}\left(v_{j}^{T} A_{k}^{T}+b_{k}^{T}\right) c_{j}
\end{array}\right]>0
$$

\section{Proof}

1 The Lyapunov function (4) is radially unbounded (see Remark 1). Therefore its level sets are closed curves [11]. Within each region $\mathcal{R}_{k}$ the level curves of the piecewise-quadratic function need to coincide with the ellipsoidal level curves $\varepsilon_{\gamma}^{k}$ of the corresponding quadratic sector. This ensures that in $\cup_{i=1}^{M} \mathcal{R}_{i}$, the $\gamma$-level sets are represented by $\cup_{k=1}^{M}\left(\varepsilon_{\gamma}^{k} \cap \mathcal{R}_{k}\right)$. To see that in fact $\varepsilon_{\gamma}=\cup_{k=1}^{M}\left(\varepsilon_{\gamma}^{k} \cap \overline{\mathcal{R}_{k}}\right)$ are the level $\gamma$-level sets of the Lyapunov function (4) wee need now only analyze what happens at the boundaries between regions. Observe that the ellipsoidal level curves for each region meet at the boundaries and form closed concatenated ellipsoidal level curves for the piecewise-quadratic function because the Lyapunov function is continuous at the boundary. Because of this fact, for all $\gamma>0$ such that $\varepsilon_{\gamma} \subseteq \mathcal{D}, \varepsilon_{\gamma}$ is a $\gamma$-level set of the Lyapunov function (4) which is a local Lyapunov function defined in $\mathcal{D}$. Therefore, as a $\gamma$-level set of a Lyapunov function, $\varepsilon_{\gamma}$ is an invariant set of the system. Moreover, Theorem 2.1 applies which leads to the conclusion and finishes the proof of this part.

$2.1(\Rightarrow)$ Assume that $\tilde{\varepsilon}_{\gamma}$ is invariant but $\tilde{\varepsilon}_{\gamma} \cap \partial_{\mathcal{D}_{j}} \cap \dot{\mathcal{D}_{j}^{+}} \neq \emptyset$ for some $j=1, \ldots, p$. This implies that $c_{j}^{T} \dot{x}_{0}>0$ for at least one point $x_{0} \in \tilde{\varepsilon}_{\gamma} \cap \partial_{\mathcal{D}_{j}}$. Thus, from Remark $4, f\left(x_{0}\right)=\dot{x}_{0} \notin \mathcal{C}_{\mathcal{D}_{j}} \Rightarrow f\left(x_{0}\right) \notin \mathcal{C}_{\tilde{\varepsilon}_{\gamma}}$, since at $x_{0}$ the two cones coincide. By Theorem 3.1, $\tilde{\varepsilon}_{\gamma}$ is not an invariant set, violating the assumption.

$2.2(\Leftarrow)$ We want to prove now that given the assumption $\tilde{\varepsilon}_{\gamma} \cap \partial_{\mathcal{D}_{j}} \cap \dot{\mathcal{D}_{j}^{+}}=\emptyset, j=1, \ldots, p$ we have $f(x) \in \mathcal{C}_{\tilde{\varepsilon}_{\gamma}}, \forall x \in \partial_{\tilde{\varepsilon}_{\gamma}}$, which guarantees invariance from Theorem 3.1. Note that $\mathcal{D} \subseteq \mathcal{X} \equiv \cup_{i=1}^{M} \overline{\mathcal{R}}_{i}$ and (as observed in 1.) $\varepsilon_{\gamma}$ is a $\gamma$-level set of the Lyapunov function (4) inside $\mathcal{D}$. Because of these facts, it suffices to check the invariance for the portions of the boundary $\partial_{\tilde{\varepsilon}_{\gamma}}$ corresponding to $x \in \partial_{\varepsilon_{\gamma}^{k}}, x \in \partial_{\mathcal{D}_{j}}$ and any region of $\partial_{\tilde{\varepsilon}_{\gamma}}$ formed by the intersection of two or more sets of the form $\varepsilon_{\gamma}^{k}$ or $\mathcal{D}_{j}$. For $x \in \partial_{\tilde{\varepsilon}_{\gamma}}$ such that $x \in \partial_{\varepsilon_{\gamma}^{k}}$ defined in (15), $f(x) \in \mathcal{C}_{\varepsilon_{\gamma}^{k}}$ is guaranteed by making the Lyapunov function negative definite since this condition implies that $\left(x-x_{c l}^{k}\right)^{T} P_{k} \dot{x}<0$. For $x \in \partial_{\tilde{\varepsilon}_{\gamma}}$ such that $x \in \partial_{\mathcal{D}_{j}}$, the assumption $\tilde{\varepsilon}_{\gamma} \cap \partial_{\mathcal{D}_{j}} \cap \dot{\mathcal{D}_{j}^{+}}=\emptyset, j=1, \ldots p$ implies $c_{j}^{T} \dot{x} \leq 0$, which in turn implies that $f(x) \in \mathcal{C}_{\mathcal{D}_{j}}$. If $x \in \partial_{\tilde{\varepsilon}_{\gamma}}$ is such that $x \in\left[\partial_{\varepsilon_{\gamma}^{k}} \cap \partial_{\mathcal{D}_{j}}\right]$ then from the above we have $f(x) \in \mathcal{C}_{\varepsilon_{\gamma}^{k}}$ and $f(x) \in \mathcal{C}_{\mathcal{D}_{j}}$ and therefore $f(x) \in\left(\mathcal{C}_{\varepsilon_{\gamma}^{k}} \cap \mathcal{C}_{\mathcal{D}_{j}}\right)$, which implies that $f(x) \in \mathcal{C}_{\tilde{\varepsilon}_{\gamma}}$. More generally this holds for any region of $\partial_{\tilde{\varepsilon}_{\gamma}}$ formed by the intersection of two or more sets (ellipsoids or sets $\mathcal{D}_{j}$ ) by repeatedly applying the above reasoning. This argument finishes this part of the proof. 
3. In the previous parts of the proof we have shown that for $\tilde{\varepsilon}_{\gamma}$ to be an invariant set, a necessary and sufficient condition is

$$
c_{j}^{T} \dot{x} \leq 0, \forall x \in \tilde{\varepsilon}_{\gamma} \cap \partial_{\mathcal{D}_{j}}, j=1, \ldots, p .
$$

However, this necessary and sufficient condition is not easy to formulate as an LMI problem. A sufficient condition for $\tilde{\varepsilon}_{\gamma}$ being an invariant set is

$$
x \in \mathcal{H}_{j} \cap \varepsilon_{\gamma} \Rightarrow c_{j}^{T} \dot{x}<0, j=1, \ldots, p .
$$

Indeed, (20) implies (19) because $\tilde{\varepsilon}_{\gamma} \subseteq \varepsilon_{\gamma}$ by definition and $\partial_{\mathcal{D}_{j}} \subseteq \mathcal{H}_{j}$ from (17). Given the definition of $\varepsilon_{\gamma}$ this sufficient condition will be relaxed to (Figure 1)

$$
x \in \mathcal{H}_{j} \cap \varepsilon_{\gamma}^{k} \Rightarrow c_{j}^{T} \dot{x}<0, \forall_{(j, k)} \mid \overline{\mathcal{R}_{k}} \cap \mathcal{H}_{j} \neq \emptyset
$$

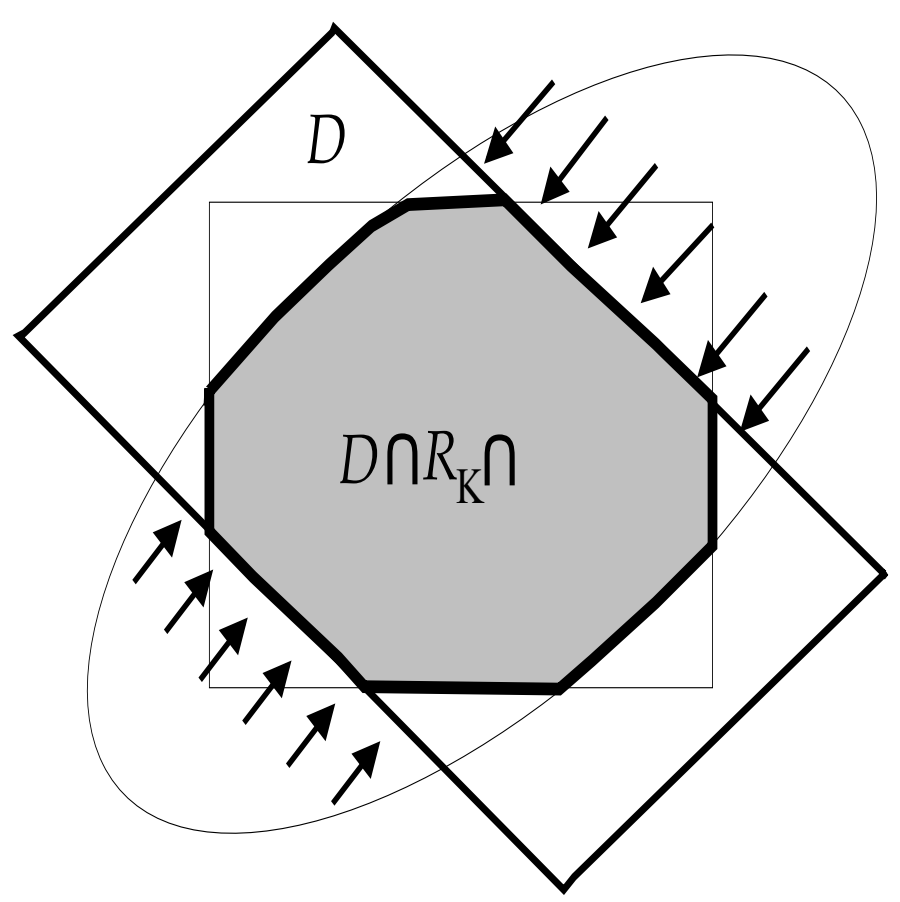

Figure 1: Invariant Regions of Lyapunov Function

Again, (21) implies (20) because $\left(\mathcal{H}_{j} \cap \varepsilon_{\gamma}^{k} \cap \overline{\mathcal{R}_{k}}\right) \subseteq\left(\mathcal{H}_{j} \cap \varepsilon_{\gamma}^{k}\right)$. Using (17) and (15) yields

$$
\begin{gathered}
x \in \mathcal{H}_{j} \cap \varepsilon_{\gamma}^{k} \Leftrightarrow \\
{\left[\begin{array}{c}
s \\
1
\end{array}\right]^{T}\left[\begin{array}{cc}
T_{j}^{T} P_{k} T_{j} & T_{j}^{T} P_{k}\left(v_{j}-x_{c l}^{k}\right) \\
(\cdot)^{T} & \left(v_{j}-x_{c l}^{k}\right)^{T} P_{k}\left(v_{j}-x_{c l}^{k}\right)+r_{k}-\gamma
\end{array}\right]\left[\begin{array}{c}
s \\
1
\end{array}\right] \leq 0 .}
\end{gathered}
$$

Using (17) yields

$$
x \in \mathcal{H}_{j} \cap\left\{x \mid c_{j}^{T} \dot{x}<0\right\} \Leftrightarrow
$$




$$
\left[\begin{array}{c}
s \\
1
\end{array}\right]^{T}\left[\begin{array}{cc}
0 & T_{j}^{T} A_{k}^{T} c_{j} \\
\left(T_{j}^{T} A_{k}^{T} c_{j}\right)^{T} & 2\left(v_{j}^{T} A_{k}^{T}+b_{k}^{T}\right) c_{j}
\end{array}\right]\left[\begin{array}{c}
s \\
1
\end{array}\right]<0 .
$$

Using (22), (23) and the $\mathcal{S}$-procedure the condition of the Theorem results, which finishes the proof.

Remark 5 Note that the necessary and sufficient condition for positively invariance from Theorem 3.1 was replaced by a sufficient condition, which is less sharp and more conservative. However, the problem was formulated as an LMI, which can be solved efficiently by convex optimization software packages.

Remark 6 Part 2 of the Theorem can be seen as a generalization to PWA systems of the results presented in [15] for state-feedback of constrained linear systems. The geometrical ideas in [15] were also generalized to relax the necessary and sufficient condition to a sufficient condition for the case of PWA systems. Unfortunately, the procedure for computing appropriate subspaces adopted in [15] to formulate the relaxation as an LMI is not straightforward to generalize to PWA systems. Therefore, the formulation of the relaxation into an LMI differs substantially from the procedure adopted in [15]. Instead of computing a subspace, the current work uses a parametric description of the boundaries of $\mathcal{D}$, as described in (17). Given that these boundaries are hyperplanes, assuming the existence of a parametric description is appropriate and it enables a simplified formulation of the LMI for the more complex case of PWA systems.

Remark 7 For a given fixed Lyapunov function, one can search for the maximum value of $\gamma$ that verifies (18) by solving the semidefinite program

$$
\begin{aligned}
\max & \gamma \\
\text { s.t. } & \epsilon_{j k}>0, \gamma>0,(18) \\
& \forall_{(j, k)} \mid \overline{\mathcal{R}_{k}} \cap \mathcal{H}_{j} \neq \emptyset
\end{aligned}
$$

Remark 8 Another interesting problem would be to find the Lyapunov function that maximizes the volume of the invariant set verifying the conditions of either Theorem 2.1 or Theorem 3.1. However, it does not seem that such problem can be formulated as a convex program.

\section{Example}

This example considers a circuit with a nonlinear resistor shown in figure 2 [5]. This nonlinear resistor model is sometimes used to approximate the behavior of a tunnel diode. With time expressed in $10^{-10}$ seconds, the inductor current in milliAmps and the capacitor voltage in Volts, the dynamics are written as

$$
\left[\begin{array}{c}
\dot{x}_{1} \\
\dot{x}_{2}
\end{array}\right]=\left[\begin{array}{rr}
-30 & -20 \\
0.05 & 0
\end{array}\right]\left[\begin{array}{l}
x_{1} \\
x_{2}
\end{array}\right]+\left[\begin{array}{c}
24 \\
-50 g\left(x_{2}\right)
\end{array}\right]+\left[\begin{array}{c}
20 \\
0
\end{array}\right] u .
$$




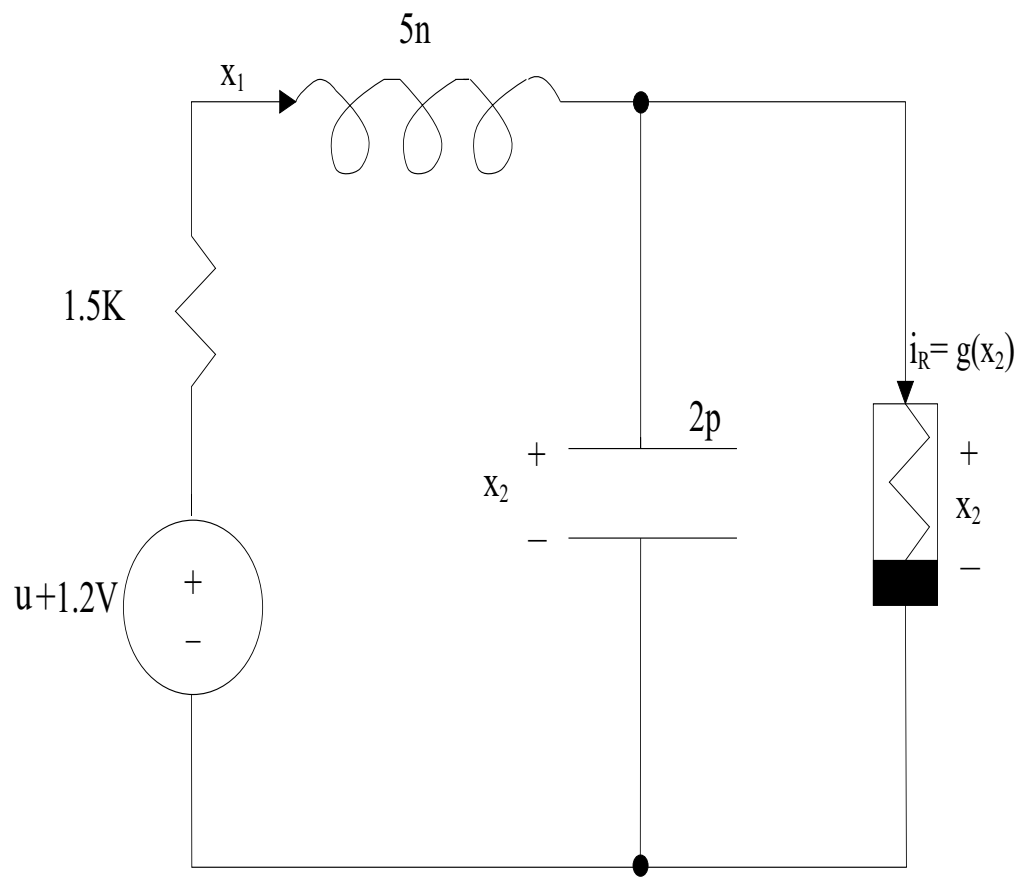

Figure 2: Invariant Regions of Lyapunov Function

Following [5], the characteristic of the nonlinear resistor $g\left(x_{2}\right)$ is defined to be the piecewise-affine function shown in figure 3 which generates the polytopic regions

$$
\mathcal{R}_{1}=\left\{x \in \mathbb{R}^{2} \mid 0<x_{2}<0.2\right\}, \quad \mathcal{R}_{2}=\left\{x \in \mathbb{R}^{2} \mid 0.2<x_{2}<0.6\right\}, \quad \mathcal{R}_{3}=\left\{x \in \mathbb{R}^{2} \mid 0.6<x_{2}<0.8\right\}
$$

Using the method explained in Section 2, a piecewise-affine state feedback controller has been designed to stabilize the open-loop equilibrium point of region $\mathcal{R}_{3}$

$$
x_{c l}=x_{o l}^{3}=\left[\begin{array}{l}
0.3714 \\
0.6429
\end{array}\right] \text {. }
$$

For the design set

$$
\mathcal{D}=\left\{x \in \mathbb{R}^{2} \mid-3 \leq x_{1} \leq 3,0 \leq x_{2} \leq 0.78\right\}
$$

the resulting controller parameters are

$$
\begin{array}{ll}
K_{1}=\left[\begin{array}{ll}
0.3523 & +1.8371
\end{array}\right], & m_{1}=0.7094 \\
K_{2}=\left[\begin{array}{ll}
0.3523 & -3.3611
\end{array}\right], & m_{2}=1.7605 \\
K_{3}=\left[\begin{array}{ll}
0.3523 & +3.0258
\end{array}\right], & m_{3}=-2.076
\end{array}
$$




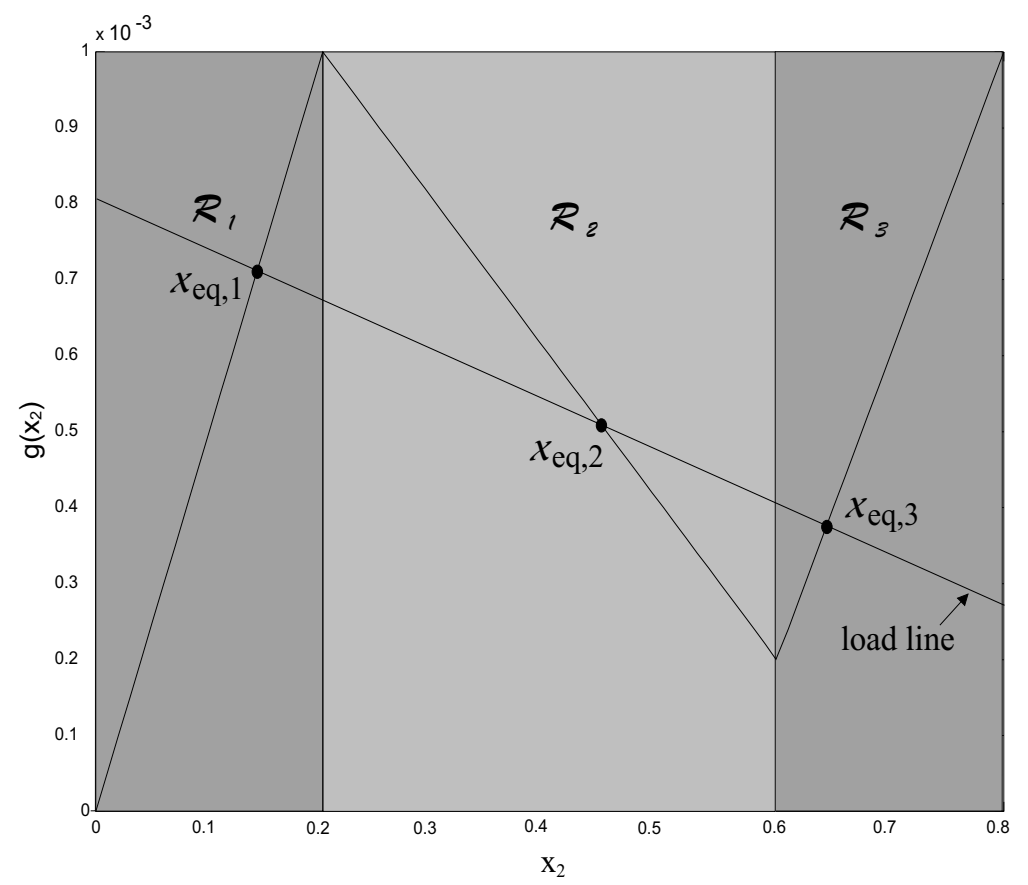

Figure 3: Nonlinear resistor characteristic.

and the Lyapunov function parameters are

$$
\begin{gathered}
P_{1}=\left[\begin{array}{cc}
1.5587 & -4.3011 \\
-4.3011 & 688.18
\end{array}\right], P_{2}=\left[\begin{array}{cc}
1.5587 & 8.8930 \\
8.8930 & 249.18
\end{array}\right], P_{3}=\left[\begin{array}{ll}
1.5587 & 19.587 \\
19.587 & 687.64
\end{array}\right], \\
q_{1}=\left[\begin{array}{c}
-4.1153 \\
-785.52
\end{array}\right], q_{2}=\left[\begin{array}{c}
-6.7541 \\
-195.6238
\end{array}\right], q_{3}=\left[\begin{array}{l}
-13.171 \\
-449.33
\end{array}\right], \\
r_{1}=365.54, \quad r_{2}=147.14, \quad r_{3}=293.74
\end{gathered}
$$

Using Theorem 2.1, exponential stability can only be proven inside the largest level set of the Lyapunov function fully contained in the design set (see figure 4). For the same fixed Lyapunov function, using Theorem 3.1 and the optimization from Remark 7, exponential stability can be proven inside intersection of the design set and the sublevel set whose boundary is the outer curve in Region $\mathcal{R}_{1}$ in figure 4 . This outer curve corresponds to the level curve of the Lyapunov function for the obtained maximum value of $\gamma=239.73$. From figure 4, it can be clearly seen that Theorem 3.1 yielded a much larger region of guaranteed exponential stability. It can also be seen that at the boundaries of the region of stability the flow points inwards, as expected. These results show one example where the conservatism of Theorem 2.1 can be greatly reduced by using the new result presented in Theorem 3.1. 


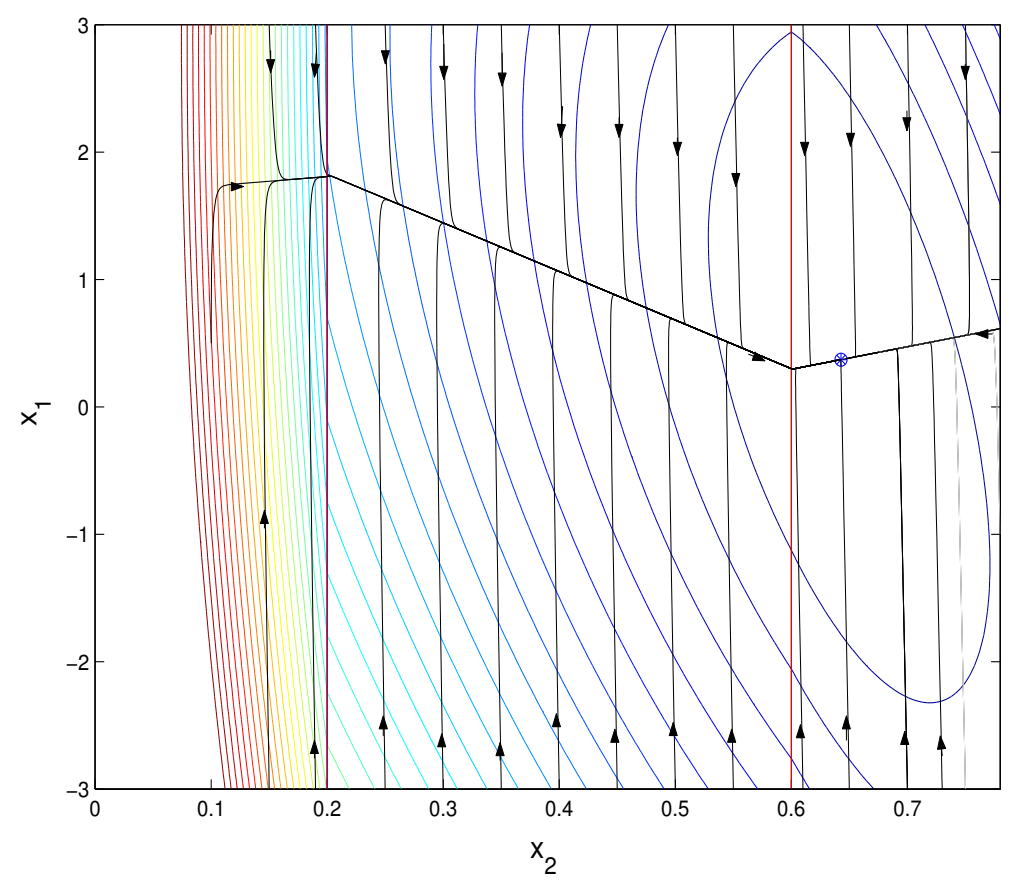

Figure 4: Level curves and regions of stability.

\section{Conclusions}

This paper presented a new stability Theorem for closed-loop PWA systems. If the conditions of the Theorem are verified then a larger region of exponential stability can be guaranteed than it would be possible using previous results. The Theorem is based on controlled invariant sets for PWA systems. Invariant sets for piecewise-affine systems were defined by extending the notion of semi-ellipsoidal invariant sets for constrained linear systems reported in previous research. A numerical example has shown a particular case for which a much larger region of guaranteed exponential stability can be obtained using the result presented in this paper instead of previous approaches.

\section{References}

[1] F. Blanchini, Set invariance in control, Automatica, vol. 35, no. 11, 1999, pp. 1747-1767.

[2] S. Boyd, L. E. Ghaoui, E. Feron, and V. Balakrishnan, Linear Matrix Inequalities in System and Control Theory, vol. 15 of Studies in Applied Mathematics, SIAM, Philadelphia, 1994.

[3] K. C. Goh, J. H. Ly, L. Turand, and M. G. Safonov, Biaffine matrix inequality properties and computational methods, in: Proceedings of the American Control Conference, 1994, pp. 850-855.

[4] P. O. Gutman and M. Cwikel, Admissible sets and feedback control for discrete-time linear dynamical systems, IEEE Transactions on Automatic Control, vol. AC-31, 1986, pp. 373-376.

[5] A. Hassibi and S. P. Boyd, Quadratic stabilization and control of piecewise-linear systems, in: Proceedings of the American Control Conference, 1998, pp. 3659-3664. 
[6] T. A. Henzinger and P.-H. Ho, A note on abstract interpretation strategies for hybrid automata, in Hybrid Systems II, Lecture Notes in Computer Science, Springer-Verlag, 1995.

[7] M. Jirstrand, Invariant sets for a class of hybrid systems, in: Proceedings of the 37th IEEE Conference on Decision and Control, 1998, pp. 3699-3704.

[8] M. Johansson and A. Rantzer, Computation of piecewise quadratic lyapunov functions for hybrid systems, IEEE Transactions on Automatic Control, vol. 43, 1998, pp. 555-559.

[9] A. A. Julius and A. J. van der Schaft, The maximal controlled invariant set of switched linear systems, in: Proceedings of the 41st IEEE Conference on Decision and Control, 2002, pp. 3174-3179.

[10] E. C. Kerrigan and D. Q. Mayne, Optimal control of constrained, piecewise affine systems with bounded disturbances, in: Proceedings of the 41st IEEE Conference on Decision and Control, 2002, pp.1552-1557.

[11] H. Khalil, Nonlinear Systems, Prentice Hall, New Jersey, 1996.

[12] H. Lin and P. J. Antsaklis, Robust controlled invariant sets for a class of uncertain hybrid systems, in: Proceedings of the 41st IEEE Conference on Decision and Control, 2002, pp. 3180-3181.

[13] M. Mukai, T. Azma, and M. Fujita, Robust receding horizon control for piecewise-linear systems based on constrained positively invariant sets, in: Proceedings of the American Control Conference, 2002, pp. 2348-2353.

[14] M. Nagumo, Über die lage der integralkurven gewöhnlicher differentialgleichungen, Proceedings of Phys.-Math Society of Japan, vol. 24, 1942, pp. 272-559.

[15] B. D. O'Dell and E. A. Misawa, Semi-ellipsoidal controlled invariant sets for constrained linear systems, in: Proceedings of the American Control Conference, 2000, pp. 1779-1783.

[16] S. Pettersson, Analysis and Design of Hybrid Systems, $\mathrm{PhD}$ thesis, Chalmers University of Technology, 1999.

[17] A. Puri and P. Varaiya, Verification of hybrid systems using abstractions, in Hybrid Systems II, Lecture Notes in Computer Science, Springer-Verlag, 1995.

[18] L. Rodrigues and J. How, Observer-based control of piecewise-affine systems, International Journal of Control, vol. 76, 2003, pp. 459-477.

[19] L. Rodrigues and J. How, Automated control design for a piecewise-affine approximation of a class of nonlinear systems, in: Proceedings of the American Control Conference, 2001, pp. 3189-3194.

[20] L. Rodrigues and J. P. How, Synthesis of piecewise-affine controllers for stabilization of nonlinear systems, Accepted for publication in 42nd IEEE Conference on Decision and Control, December 2003.

[21] L. Rodrigues, Dynamic Output Feedback Controller Synthesis for Piecewise-Affine Systems, PhD thesis, Stanford University, 2002.

[22] J. Roll, Robust verification of piecewise affine systems, in: IFAC World Congress, 2002.

[23] O. Shakernia, G. J. Pappas, and S. Sastry, Semidecidable controller synthesis for classes of linear hybrid systems, in: Proceedings of the 39th IEEE Conference on Decision and Control, 2000, pp. 1834-1839.

[24] R. Vidal, S. Schaffert, O. Shakernia, J. Lygeros, and S. Sastry, Decidable and semi-decidable controller synthesis for classes of discrete time hybrid systems, in: Proceedings of the 39th IEEE Conference on Decision and Control, 2001, pp. 1243-1248. 